\title{
Correlação entre incontinência urinária, disfunção sexual e avaliação subjetiva da contração muscular perineal em idosas fisicamente ativas
}

\author{
Urinary incontinence and female sexual dysfunction in physically active older women
}

Correlación entre incontinencia urinaria, disfunción sexual y evaluación subjetiva de la contracción del músculo perineal en mujeres ancianas físicamente activas

Giovana Zarpellon Mazo', Keyla Mara dos Santos ${ }^{2}$, Caroline Silva de Freitas ${ }^{3}$, Adriana Cielo ${ }^{4}$,
Melissa Medeiros Braz ${ }^{5}$, Hedioneia Maria Foletto Pivetta

RESUMO I Incontinência urinária (IU) e disfunção sexual feminina (DSF) são disfunções que afetam a qualidade de vida. Com a mudança na pirâmide etária brasileira, a prevalência de tais disfunções tende a aumentar. O objetivo deste estudo foi estabelecer a relação entre a IU, a DSF e a contração muscular perineal em idosas ativas fisicamente. Trata-se de estudo transversal, do qual participaram 35 idosas $\geq 60$ anos. Inicialmente foram aplicados a ficha diagnóstica e os questionários International Consultation on Incontinence Questionnaire - Short Form (ICIQ-UI-SF) e Female Sexual Function Index (FSFI). Em seguida foram avaliados os músculos do assoalho pélvico (MAP) por meio do toque vaginal, de acordo com o que propõe o esquema perfect. Entre as idosas, 20\% apresentaram ambas as disfunções dos MAP. No total, 17 (48,6\%) apresentavam IU e 16 (45,7\%) DSF. Observou-se diferença significativa no número de partos vaginais $(p=0,028)$ e no item rapidez do esquema perfect ( $p=0,033)$ entre as idosas com e sem DSF. Não houve diferença estatística entre os grupos com e sem IU. A análise apontou uma correlação inversamente proporcional entre a gravidade da IU e a função sexual nas idosas incontinentes ( $p=0,008 ; r=-0,622)$, de forma que, para cada aumento na gravidade da IU pelo ICIQ-SF, diminuiu-se 0,507 de função sexual avaliada pelo FSFI ( $p=0,034 ; r=0,516)$. Há uma associação entre IU e DSF em idosas fisicamente ativas. Algumas idosas apresentam ambas as disfunções dos MAP, apesar de serem ativas fisicamente. Dessa forma, é necessário reforçar a importância de avaliações minuciosas, da atividade física orientada e da atuação multiprofissional em saúde.

Descritores | Atividade Física; Assoalho Pélvico; Pessoa Idosa; Saúde Sexual; Incontinência Urinária.

\begin{abstract}
I Urinary incontinence (UI) and female sexual dysfunction (FSD) are disorders that affect quality of life. With the change in the Brazilian age pyramid, the prevalence of such disorders tends to increase. The aim of the present study was to correlate the relationship between UI, FSD and the functionality of the pelvic floor muscles (PFM) in physically active older women. This is a cross-sectional study, in which 35 older women $\geq 60$ years old participated. Initially, the diagnostic form, the International Consultation on Incontinence Questionnaire - Short form (ICIQ-UI-SF) and Female Sexual Function Index (FSFI) were applied. Then, the PFM were evaluated by vaginal touch, following what is proposed in the perfect scheme. Among older women, 20\% had both PFM dysfunctions. In total, 17 (48.6\%) had UI and 16 (45.7\%) FSD. There was a significant difference in the number of vaginal deliveries ( $\mathrm{p}=0.028$ ) and in the perfect schedule $(p=0.033)$ between older women with and without FSD. There was no statistical difference between the groups with and without UI. The analysis showed an inversely proportional
\end{abstract}

\footnotetext{
Universidade do Estado de Santa Catarina (Udesc) - Florianópolis (SC), Brasil. E-mail: giovana.mazo@udesc.br. Orcid: 0000-0002-7813-5592

2Universidade do Estado de Santa Catarina (Udesc) - Florianópolis (SC), Brasil. E-mail: keylasantosfisio@gmail.com. Orcid: 0000-0002-7868-9798

3 Universidade Federal de Santa Maria (UFSM) - Santa Maria (RS), Brasil. E-mail: carolfisio.88@gmail.com. Orcid: 0000-0002-3715-6717 ${ }^{4}$ Universidade Federal de Santa Maria (UFSM) - Santa Maria (RS), Brasil. E-mail: adrianacielo025@hotmail.com.

Orcid: 0000-0002-0597-2082

${ }^{5}$ Universidade Federal de Santa Maria (UFSM) - Santa Maria (RS), Brasil. E-mail: melissabraz@hotmail.com. Orcid: 0000-0002-9138-0656 6Universidade Federal de Santa Maria (UFSM) - Santa Maria (RS), Brasil. E-mail: hedioneia@yahoo.com.br. Orcid: 0000-0003-2713-401X
} 
correlation between $\mathrm{UI}$ severity and sexual function in incontinent older women. ( $p=0.008 ; r=-0.622)$. Since, for each increase in UI severity by the ICIQ-SF, 0.577 of sexual function assessed by the FSFI is decreased ( $p=0.034 ; r=0.516)$. There is an association between $\mathrm{UI}$ and FSD in physically active older women. Some older women have both PFM dysfunctions despite being physically active. Thus, it is important to reinforce the importance of thorough evaluations, oriented physical activity and multiprofessional action in health.

Keywords I Physical Activity; Pelvic Floor; Older Population; Sexual Health; Urinary Incontinence.

RESUMEN I La incontinencia urinaria (IU) y la disfunción sexual femenina (DSF) son trastornos que afectan la calidad de vida de las personas. Con el cambio en la pirámide de edad brasileña se aumentará la prevalencia de tales disfunciones. El objetivo de este estudio fue establecer la relación entre la IU, la DSF y la contracción del músculo perineal en mujeres mayores físicamente activas. Este es un estudio transversal, en el que participaron 35 mujeres $\geq 60$ años. Inicialmente se aplicó el formulario de diagnóstico y los cuestionarios International Consultation on Incontinence Questionnaire - Short
Form (ICIQ-UI-SF) e Índice de Función Sexual Femenina (FSFI). Luego, se evaluó la musculatura del piso pélvico (MAP) mediante toque vaginal siguiendo lo que propone el esquema perfect. Entre las ancianas, el 20\% tenía ambas disfunciones de MAP. En total, 17 (48,6\%) tenía IU, y 16 (45,7\%) DSF. Hubo una diferencia significativa en el número de partos vaginales $(p=0,028)$ y en el ítem rapidez del esquema perfect $(p=0,033)$ entre mujeres ancianas con y sin DSF. No hubo diferencia estadística entre los grupos con y sin IU. El análisis mostró una correlación inversamente proporcional entre la gravedad de la IU y la función sexual en mujeres ancianas con incontinencia ( $p=0,008 ; r=-0,622)$, de modo que por cada aumento en la gravedad de la IU por el ICIQ-SF se disminuyó 0,507 de función sexual evaluada por el FSFI ( $p=0,034 ; r=0,516)$. Existe una asociación entre la IU y la DSF en mujeres ancianas físicamente activas. Algunas ancianas tuvieron ambas disfunciones del MAP a pesar de ser físicamente activas. Por lo tanto, es necesario reforzar la importancia de las evaluaciones detalladas, la actividad física orientada y la acción multiprofesional en salud.

Palabras clave | Actividad Física; Suelo Pélvico; Anciano; Salud Sexual; Incontinencia Urinaria.

\section{INTRODUÇÃO}

Nos últimos anos o Brasil vem enfrentando mudanças importantes na pirâmide etária, acarretando aumento no número de pessoas idosas no país. Dessa maneira, é fundamental dar mais atenção às disfunções crônicas e de funcionalidade dos músculos do assoalho pélvico (MAP), relacionadas com o avançar da idade ${ }^{1}$.

No que diz respeito às idosas, as disfunções dos MAP são motivo de grande preocupação ${ }^{2-4}$. Dentre as disfunções, destacam-se a incontinência urinária (IU) e a disfunção sexual feminina (DSF), por causa prevalência acentuada e impacto negativo na qualidade de vida ${ }^{5}$. A IU é caracterizada pela International Continence Society (ICS) como qualquer perda involuntária de urina ${ }^{6}$, sendo prevalente entre idosas brasileiras com mais de 60 anos $(26,2 \%)^{7}$. A DSF caracterizase pelo déficit em uma das fases do ciclo de resposta sexual feminino, que consiste em: desejo/excitação, platô, orgasmo e resolução ${ }^{4}$. Novos modelos caracterizam a resposta sexual feminina como não linear, sugerindo que para a mulher haveria um componente subjetivo, contradizendo as teorias tradicionais de linearidade. No estudo de Wolpe et al. ${ }^{4}$ constatou-se que a prevalência de DSF no Brasil é de aproximadamente $67,7 \%$, sendo maior em mulheres de meia idade, em decorrência dos sintomas do período climatérico.
Sabe-se que o envelhecimento provoca uma série de alterações fisiológicas no organismo feminino ${ }^{8}$. Destacamse a atrofia muscular e a substituição de tecido muscular por adiposo ${ }^{8}$. Tais alterações acometem também os MAP, acarretando diminuição da força de contração muscular e podendo levar a disfunções como a DSF e a IU' ${ }^{9}$.

Por outro lado, estudos apontam que a atividade física gera inúmeros benefícios para a população idosa, incluindo a melhora da funcionalidade dos MAP. No estudo de Virtuoso, Mazo e Menezes ${ }^{9}$ foram avaliadas 39 mulheres com idade superior a 60 anos, divididas em dois grupos - grupo praticantes (GP), no qual as mulheres eram praticantes de atividades físicas, e grupo não praticantes (GNP), no qual as mulheres não eram praticantes de atividades físicas. Os autores constataram que as idosas do GP apresentaram maior número de repetições $(\mathrm{p}=0,008)$ e maior rapidez $(\mathrm{p}=0,022)$ nas contrações dos MAP, além de maior força muscular na perineometria (fibras lentas $(\mathrm{p}=0,050)$ e rápidas $(\mathrm{p}=0,008)$ ).

Diante da necessidade de compreender as disfunções dos MAP que acometem a mulher idosa fisicamente ativa, este estudo fornece dados que poderão influenciar o ponto de vista clínico, levando o profissional a investigar a presença de disfunções relacionadas aos MAP. Além disso, do ponto de vista científico, este estudo levanta questões 
para pesquisas futuras e, do ponto de vista social, auxilia no tratamento das idosas.

Este estudo visou estabelecer a relação entre a IU, a DSF e a contração muscular perineal em mulheres idosas ativas fisicamente. Como objetivos secundários buscou-se verificar se existe diferença na contração dos MAP e na IU entre idosas fisicamente ativas com e sem DS e identificar se há diferença na contração muscular perineal e na função sexual entre idosas fisicamente ativas com e sem IU.

\section{METODOLOGIA}

Trata-se de estudo transversal que visou estabelecer a relação entre a IU, a DSF e a contração dos MAP em idosas fisicamente ativas. A população foi composta por idosas com mais de 60 anos que participam do Núcleo Integrado de Estudos e Apoio à Terceira Idade (Nieati) do Centro de Educação Física e Desportos (CEFD) da Universidade Federal de Santa Maria (UFSM), em Santa Maria, Rio Grande do Sul. Uma amostra de 34 mulheres foi estimada para obter um nível de significância de $5 \%$ e poder de 95\%, com base no estudo de Virtuoso, Mazo e Menezes ${ }^{9}$. O cálculo amostral foi realizado por meio do software Gpower, versão 3.1. Foi utilizada para as correlações entre variáveis uma análise a priori considerando um tamanho do efeito de 0,5, nível de significância de 0,05 e poder de 0,95. Para comparações entre variáveis foi utilizada uma análise de sensibilidade, considerando o atual número amostral com um poder de 0,80 , e nível de significância de 0,05. O tamanho do efeito foi de 0,97 .

Para concretizar o estudo foram adotados como critérios de inclusão: mulheres com 60 anos ou mais, participantes do Nieati-CEFD-UFSM; com capacidade cognitiva preservada; independentes funcionalmente; ativas sexualmente; que aceitassem fazer uso do acelerômetro por pelo menos cinco dias na semana, com uso mínimo de 10 horas por dia. Como critérios de exclusão foram adotados: idosas que estavam realizando terapia de reposição hormonal; tosse crônica referida; deficiência física ou sequelas de patologias neurológicas; tratamento medicamentoso da IU; sexualmente inativas; e mulheres que realizassem atividade física na água (natação, hidroginástica, hidroterapia), pois isso inviabilizaria o uso do acelerômetro.

Para verificar a função cognitiva foi utilizado o Miniexame do Estado Mental (Meem). Idosas que não apresentaram comprometimento cognitivo foram incluídas no estudo ( $>25$ pontos). A escala de Katz foi utilizada para avaliar as atividades da vida diária (AVD) e o nível de dependência.
Para definir o nível de atividade física (AF), as idosas utilizaram um acelerômetro por pelo menos cinco dias, com uso mínimo de 10 horas por dia, previamente à coleta de dados. $O$ ponto de corte foi utilizado com base nos valores de referência de intensidade da $\mathrm{AF}$ moderada diária, igual ou superior a 1.041 counts por minuto. Após a aplicação dos critérios de inclusão/exclusão foram recrutadas 35 idosas (Figura 1). As participantes assinaram um Termo de Consentimento Livre e Esclarecido (TCLE).

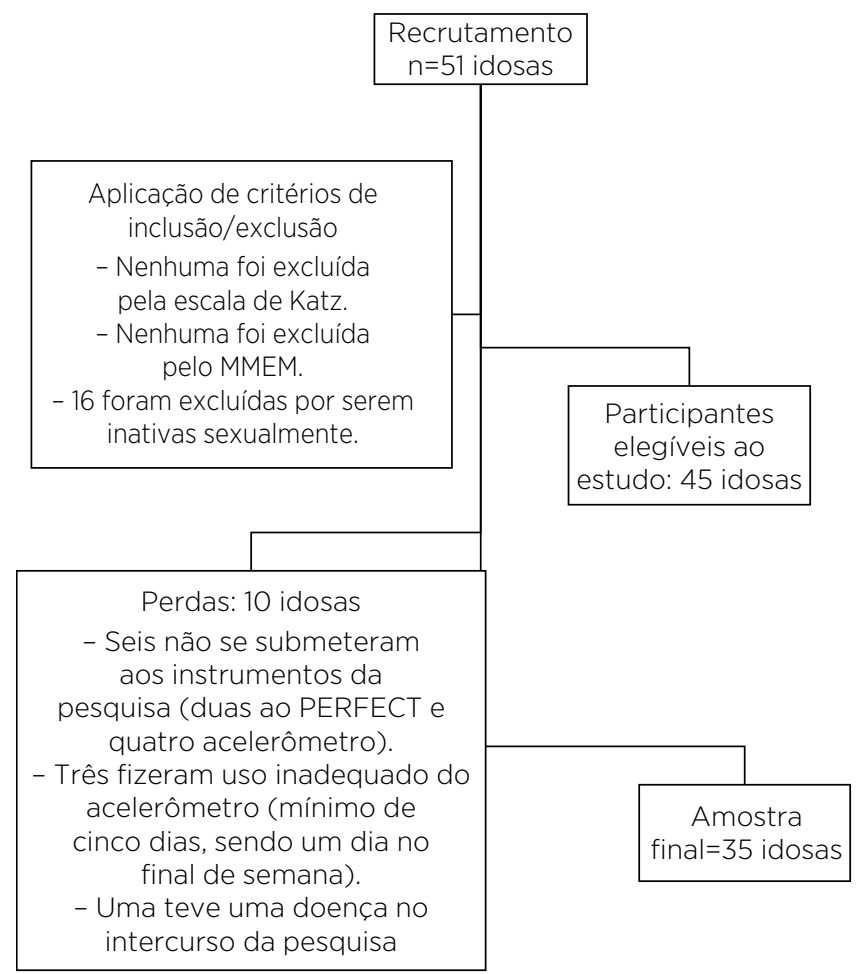

Figura 1. Fluxograma referente ao recrutamento do estudo

A coleta de dados foi realizada em uma clínica de fisioterapia climatizada em $24^{\circ} \mathrm{C}$. As pesquisadoras foram treinadas e realizaram as avaliações com cegamento: uma delas aplicou entrevistas e a outra avaliou os MAP.

Primeiramente foi aplicada uma ficha diagnóstica composta por 20 questões distribuídas em quatro blocos (Bloco 1: dados de identificação; Bloco 2: características sociodemográficas; Bloco 3: condições de saúde; Bloco 4: atividade física). A ficha foi adaptada contendo dados uroginecológicos das pacientes.

Em seguida foi aplicado o questionário International Consultation on Incontinence Questionnaire - Short Form (ICIQ-UI-SF), que avalia frequência, gravidade e impacto da IU na qualidade de vida. O escore varia de 0 a 21; quanto maior a pontuação maior é a gravidade da $\mathrm{IU}^{10}$.

Para avaliar a função sexual foi aplicado o questionário Female Sexual Function Index (FSFI), composto por 
19 questões divididas em seis domínios: desejo sexual, excitação sexual, lubrificação vaginal, orgasmo, satisfação sexual e dor ${ }^{11}$. Cada questão podia ser pontuada de 0 a 5. A pontuação final ocorria pela soma dos escores ponderados, sendo no máximo 36 . Valores iguais ou abaixo de 26,55 são sugestivos de DSF ${ }^{11}$.

Após aplicação dos questionários, as voluntárias foram convidadas a realizar uma avaliação física dos MAP pelo esquema perfect ${ }^{12}$. Essa avaliação, realizada por fisioterapeuta capacitada, ocorreu com a paciente em litotomia, na qual foi feita a palpação intravaginal, introduzindo-se o $2^{\circ}$ e $3^{\circ}$ dedos de $3-4 \mathrm{~cm}$ no intróito vaginal, com a mão devidamente enluvada e untada com gel lubrificante à base de água para diminuir o desconforto com o procedimento.

No esquema perfect a força muscular é simbolizada por "P”, representando power, e é graduada de acordo com a escala de Oxford, de zero a cinco, além de avaliar a presença e intensidade da contração muscular voluntária (CMV). A resistência muscular é representada por "E" (endurance) e corresponde ao tempo de contração voluntária sustentada. Como resultado, registra-se o tempo de sustentação da contração muscular (no máximo 10 segundos). A repetição das contrações sustentadas (fibras lentas) também é registrada, sendo representada pelo "R" (repetition). Registram-se as contrações consideradas satisfatórias (de cinco segundos) que a mulher consegue realizar após um período de repouso de quatro segundos entre elas (no máximo 10 repetições). As contrações das fibras rápidas são representadas pelo " $F$ " (fast). Verifica-se o número de contrações rápidas de um segundo sem que haja comprometimento da intensidade (no máximo 10 vezes). $\mathrm{Na}$ avaliação buscou-se isolar a contração dos MAP mediante orientação, comando verbal ("segure a urina com movimento para dentro e para cima"; "relaxe as pernas, glúteos e abdômen") e estímulo proprioceptivo. O estudo de Laycock e Jerwood ${ }^{13}$ aponta que o esquema perfect demonstrou confiabilidade e validade como ferramenta de avaliação.

A análise estatística foi realizada por meio do Statistical Package for Social Sciences (SPSS), versão 20.0. A avaliação da normalidade dos dados foi realizada pelo teste de Shapiro Wilk. O teste de Mann-Whitney foi utilizado para comparar os grupos com e sem IU, assim como os grupos com e sem DSF, nas variáveis obstétricas, urinárias, de função sexual e esquema perfect. Para verificar a relação entre a gravidade da IU e a função sexual foi utilizado o teste de correlação de Spearman. Além disso, analisou-se a regressão linear simples para verificar a influência da IU na função sexual, adotando um nível de significância de $5 \%(p<0,05)$. A correção de Bonferroni foi utilizada a fim de reduzir as chances de erro tipo I, utilizando-se $\mathrm{p}<0,025$. As idosas que apresentaram simultaneamente IU e DSF não foram excluídas das análises estatísticas.

\section{RESULTADOS}

Com relação às 35 idosas avaliadas, observou-se que $17(48,6 \%)$ apresentavam IU, enquanto 16 (45,7\%) apresentavam DSF. Ademais, os resultados apontam que $20 \%$ das idosas $(n=7)$ tinham ambas as disfunções dos MAP, simultaneamente.

A Tabela 1 mostra dados obstétricos, de IU e de contração dos MAP em relação às idosas com e sem DSF. Houve diferença significativa entre os grupos no número de partos vaginais, sendo o grupo sem DSF o que obteve a maior média $(\mathrm{p}=0,028)$. No componente do esquema perfect, denominado rapidez, que representa a contração de fibras musculares de contração rápida dos MAP, o grupo com DSF apresentou maior média ( $\mathrm{p}=0,033)$ (Tabela 1$)$.

Tabela 1. Comparação dos grupos com DSF e sem DSF em relação a dados obstétricos, de perda urinária e contração muscular perineal

\begin{tabular}{|c|c|c|c|}
\hline & Com DSF & Sem DSF & \multirow{2}{*}{ Valor de $p$} \\
\hline & Média $\pm D P$ & Média $\pm D P$ & \\
\hline Idade & $64,12 \pm 4,5$ & $66,89 \pm 5,1$ & 0,103 \\
\hline Peso & $72,19 \pm 10,9$ & $72,95 \pm 13,8$ & 0,947 \\
\hline \multicolumn{4}{|l|}{ Dados obstétricos } \\
\hline Gestações & $2,44 \pm 1,3$ & $3,11 \pm 2,0$ & 0,253 \\
\hline Partos & $2,19 \pm 1,2$ & $2,26 \pm 1,6$ & 0,697 \\
\hline Abortos & $0,25 \pm 0,7$ & $0,53 \pm 0,7$ & 0,130 \\
\hline Cesáreas & $1,00 \pm 1,2$ & $0,32 \pm 0,5$ & 0,121 \\
\hline Partos vaginais & $1,19 \pm 1,2$ & $2,42 \pm 1,7$ & $0,028^{* *}$ \\
\hline $\begin{array}{l}\text { Peso do maior } \\
\text { recém-nascido }\end{array}$ & $3,31 \pm 1,1$ & $2,89 \pm 1,6$ & 0,659 \\
\hline \multicolumn{4}{|l|}{ ICIQ-SF } \\
\hline $\begin{array}{l}\text { Frequência de } \\
\text { perda urinária }\end{array}$ & $1,38 \pm 1,8$ & $1,16 \pm 1,5$ & 0,914 \\
\hline $\begin{array}{l}\text { Quantidade de } \\
\text { perda urinária }\end{array}$ & $1,37 \pm 1,8$ & $1,32 \pm 1,4$ & 0,813 \\
\hline $\begin{array}{l}\text { Impacto da IU } \\
\text { na QV }\end{array}$ & $3,00 \pm 3,8$ & $1,26 \pm 1,6$ & 0,330 \\
\hline Gravidade da IU & $5,69 \pm 7,1$ & $3,68 \pm 3,9$ & 0,533 \\
\hline \multicolumn{4}{|l|}{ Esquema perfect } \\
\hline Força & $3,13 \pm 1,5$ & $2,47 \pm 1,4$ & 0,165 \\
\hline Manutenção & $2,50 \pm 1,2$ & $2,42 \pm 2,0$ & 0,685 \\
\hline Repetição & $3,88 \pm 2,2$ & $2,58 \pm 1,9$ & 0,111 \\
\hline Rapidez & $6,13 \pm 3,4$ & $3,63 \pm 2,6$ & $0,033^{*}$ \\
\hline
\end{tabular}

*Diferença estatisticamente significativa considerando $p<0,05$. "Diferença estatisticamente significativa considerando a correção de Bonferroni $(p<0,025)$. 
A Tabela 2 apresenta os dados obstétricos, de função sexual e de contração dos MAP em relação às idosas com e sem IU. Nota-se que não houve diferença entre os grupos.

Tabela 2. Comparação dos grupos com e sem IU em relação a dados obstétricos, de função sexual e contração muscular perineal

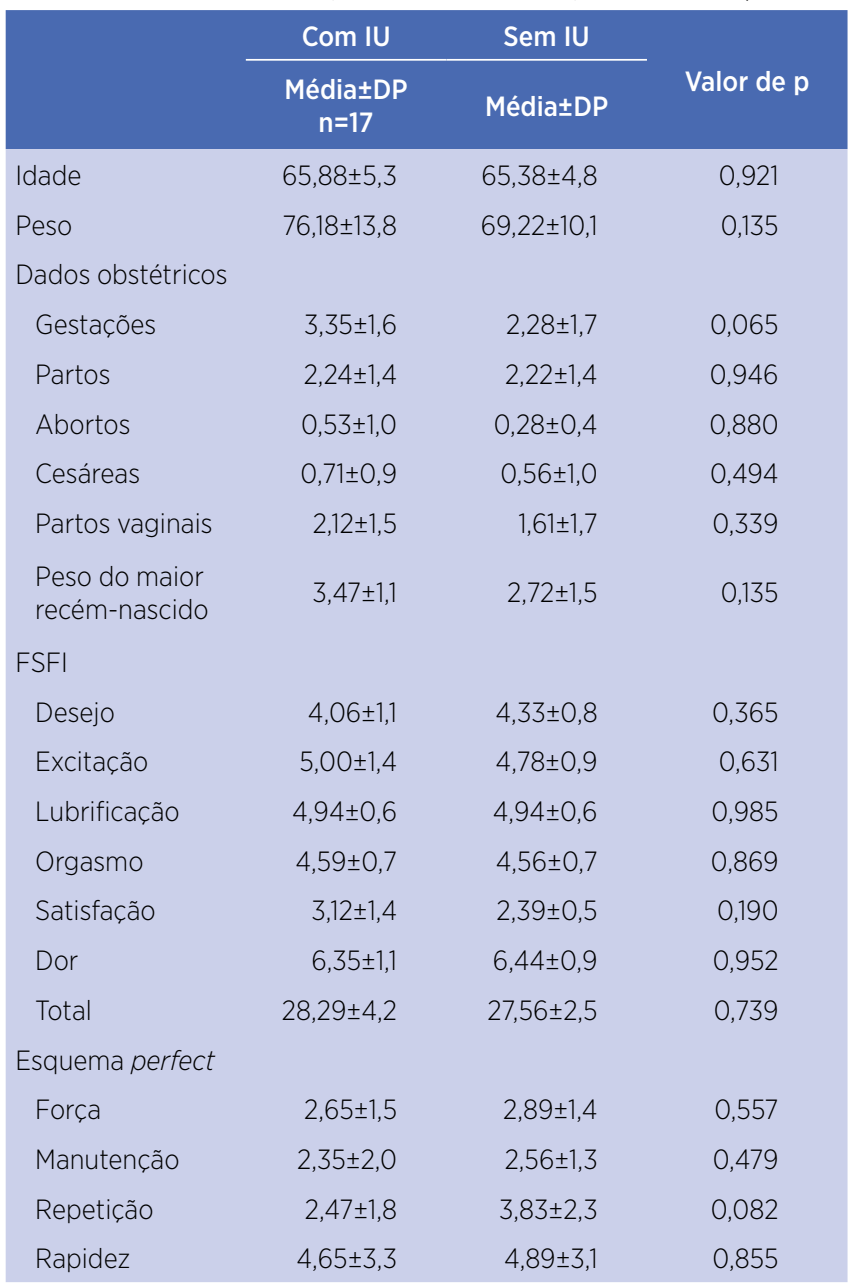

Apesar de não haver resultados significativos, ao se observar a Tabela 2 é possível notar que as mulheres com IU apresentavam uma tendência de menores resultados em relação ao esquema perfect, menor escore total do questionário FSFI de função sexual e maior número de gestações do que as idosas do grupo sem IU.

Com relação às idosas com IU, observa-se na Figura 2 que, à medida que a pontuação no questionário FSFI aumenta, diminui-se a gravidade da IU. Esse resultado sugere uma correlação entre as disfunções: quanto maior a gravidade da IU, pior a função sexual das idosas. O teste de correlação reforça esse resultado $(p=0,008 ; r=-0,622)$. De acordo com a análise de regressão linear, para cada aumento na gravidade da IU pelo ICIQ-SF, diminui-se 0,507 de função sexual avaliada pelo FSFI ( $p=0,034 ; r=0,516)$.

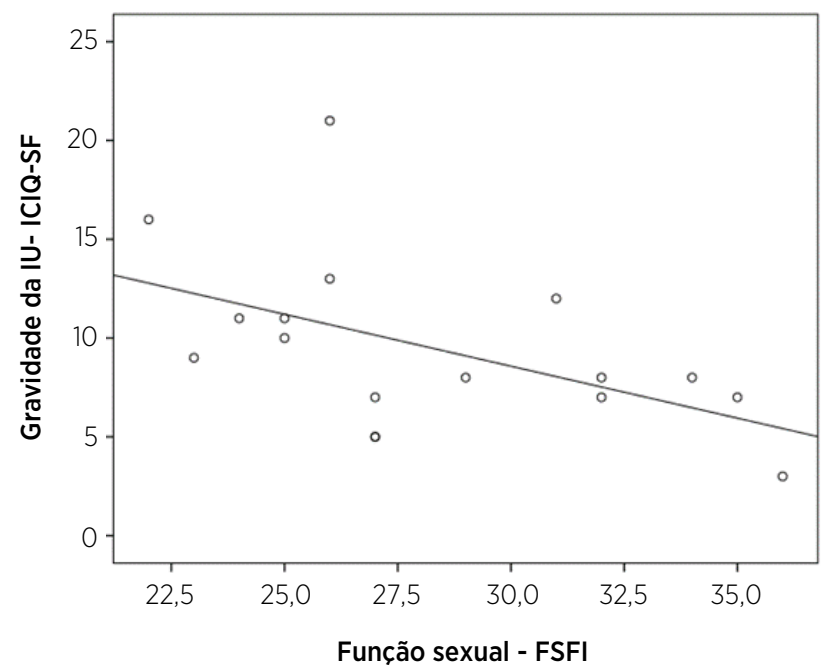

Figura 2. Correlação inversamente proporcional entre a gravidade da IU e a função sexual nas idosas incontinentes

\section{DISCUSSÃO}

Este estudo visou estabelecer a relação entre a IU, a DSF e a contração dos MAP em mulheres idosas ativas fisicamente. Os resultados mostram alta frequência das disfunções dos MAP, de forma que algumas idosas apresentaram-nas simultaneamente (20\%). Houve diferença significativa entre idosas com e sem DSF no número de partos vaginais e no item denominado rapidez do esquema perfect. Não houve diferença estatística entre idosas com e sem IU. No entanto, as idosas com IU apresentaram resultado inferior no esquema perfect e na função sexual, além de maior número de gestações do que as idosas sem IU.Verificou-se, no gráfico de dispersão, uma correlação inversamente proporcional entre a função sexual e a gravidade da IU, reforçada pelo teste de correlação e pela análise de regressão linear.

$\mathrm{O}$ avançar da idade é um dos fatores de risco mais citados para disfunções dos MAP. Esse fato está associado à diminuição dos níveis de estrógeno, à diminuição da capacidade da bexiga, que passa de 500-600 ml para 250$300 \mathrm{ml}$ em mulheres idosas, ao aumento das doenças crônicas nas faixas etárias maiores e ao aumento do índice de massa corpórea. Além da idade, a multiparidade também é considerada um importante fator de risco. Segundo Zeleke et al. ${ }^{14}$, a proporção de mulheres com um ou mais distúrbios dos MAP aumenta com a paridade. Ou seja, mulheres multíparas têm maiores chances de desenvolver disfunções dos MAP. A proporção encontrada é de 34,6\% (IC 95\%, 7,8-11,7\%) para mulheres nulíparas, $45,3 \%$ (IC 95\%, 40,3-59,1\%) para mulheres que tiveram 
de um a dois partos e 52,1\% (IC 95\%, 48,3-55,8\%) para mulheres que tiveram mais de três partos. No entanto, a alta frequência de disfunções não era um resultado esperado neste estudo, pois as idosas participantes eram ativas fisicamente.

Segundo Virtuoso, Menezes e Mazo ${ }^{15}$, o uso de diuréticos e histórico familiar positivo são os principais fatores de risco para desenvolvimento de IU em idosas praticantes de atividade física. Estudos também apontam que alguns fatores estabelecidos na literatura, como o parto e o sobrepeso, não aumentam as chances de IU no caso de uma população de idosas ativas ${ }^{16}$, e que a paridade, via de parto ou realização de episiotomia não são fatores de risco para IU entre a população idosa ${ }^{7}$. Contudo, as idosas com IU deste estudo apresentaram maior número de gestações do que as idosas sem IU, além de pior contração muscular perineal e menor escore de função sexual.

Neste estudo as mulheres sem DSF apresentaram maior número de partos normais em relação às mulheres com DSF. Esse resultado salienta que o parto vaginal, além de ter inúmeros benefícios para a mãe e para o recém-nascido, não parece predispor a mulher idosa à disfunção dos MAP. Porém, esse resultado vai de encontro aos achados da literatura. De acordo com o estudo de Zizzi et al. ${ }^{17}$, a força muscular do AP está diretamente relacionada ao tipo de parto. No estudo, as mulheres que realizaram parto vaginal tiveram menores valores de contração muscular perineal do que as que realizaram o parto cesáreo.

Além disso, as idosas com DSF apresentaram maior número de contrações rápidas (fast) no esquema perfect. No entanto, sabe-se que os MAP são constituídos de 70\% de fibras do tipo I (fibras de contração lenta) e apenas $30 \%$ de fibras do tipo II (fibras de contração rápida) ${ }^{18}$. Dessa forma, pode-se inferir que um déficit nas fibras de contração rápida, no caso das idosas deste estudo, não seria prejudicial à função sexual, pois existem em menor número na musculatura dos MAP. Da mesma forma, a melhor pontuação nas contrações rápidas não foi um fator de proteção à DSF.

Quanto à IU, sabe-se que é uma disfunção que gera um problema social e higiênico importante, sendo considerada um problema de saúde pública ${ }^{19}$. O resultado deste estudo aponta uma correlação inversamente proporcional entre a gravidade da IU e a função sexual, indicando que, quanto mais grave for a IU, maior é a chance de a idosa desenvolver disfunções sexuais. A gravidade da IU é avaliada pela frequência de perdas urinárias, quantidade de perdas e impacto da IU na qualidade de vida. Por sua vez, a disfunção sexual é definida como um déficit recorrente em qualquer uma das fases do ciclo de resposta sexual feminina ${ }^{4}$.

Outros estudos encontraram essa associação em outras populações ${ }^{20,21}$. No estudo de $\mathrm{Su}$, Sun e Jiann ${ }^{21}$ foram entrevistadas 1.450 mulheres com idade de 34,6 $\pm 8,4$ anos. Como resultado, foi verificada a associação da DSF com os três principais tipos de IU: a IU de esforço (OR: 1,6; IC: 1,1-2,3; $<<0,05)$, de urgência (OR: 1,8; IC: 1,0$3,3 ; \mathrm{p}<0,05)$ e a mista (OR: 1,6 ; IC: $1,0-2,4 ; \mathrm{p}<0,05)$. A disfunção mais comum, apresentada em todos os tipos de IU, foi no domínio do desejo sexual.

Pode-se cogitar que, além das semelhanças anatômicas, embriológicas, funcionais e endócrinas, o impacto psicológico da IU, como a vergonha, o desconforto, o medo de perder urina durante o coito, a ansiedade e a perda de autoconfiança podem explicar a associação com a DSF. Segundo alguns autores, a IU pode desencadear problemas como a perda de urina durante o coito (incontinência do coito), perdas noturnas associadas à urgência e medo de urinar na cama ${ }^{22}$. O medo de cheirar mal e a IU durante o coito estão associados à alteração da imagem e da autoestima, responsáveis pela baixa frequência de atividade sexual entre as mulheres incontinentes ${ }^{23}$.

O estudo de Visser et al. ${ }^{24}$ aponta que na população idosa a ocorrência de incontinência urinária também repercute negativamente na sexualidade. Uma pesquisa transversal foi realizada com 350 mulheres com idade superior a 55 anos. De todas as participantes com um parceiro, 68\% (IC 95\%: 61-74) eram sexualmente ativas. Destas, 25\% (IC 95\%: 17-35) se sentiam evitadas na atividade sexual por causa da IU. A perda de urina durante o ato sexual estava presente em 26\% (IC 95\%: 19-34) ${ }^{24}$ dos casos. Tais resultados demonstram que o impacto na função sexual deve ser considerado na abordagem da IU em mulheres mais velhas.

No que tange ao tratamento da IU, estudos têm demonstrado os benefícios do treinamento dos músculos do assoalho pélvico (TMAP) na redução da perda de urina e na melhora da função sexual ${ }^{25}$. No entanto, sabese que a perda de urina ainda constitui um tabu, gerando sentimentos de vergonha, frustração e desconforto que impedem muitas mulheres de iniciarem o tratamento ou mesmo de falar com os profissionais de saúde sobre o problema em questão.

Como limitações deste estudo, aponta-se que, no caso de assuntos como atividade sexual e IU, há sempre alguma insegurança que deve ser levada em consideração em relação aos dados adquiridos, pois é possível que 
respostas socialmente desejáveis sejam dadas pelas idosas. No entanto, ao fazer as perguntas sobre sexualidade por meio de questionários confiáveis e validados (e não por meio de entrevista), buscou-se reduzir esse risco tanto quanto possível. Este estudo apresentou uma avaliação profunda e fidedigna, por meio de instrumentos amplamente reconhecidos, o que torna os resultados passíveis de serem replicados.

\section{CONCLUSÃO}

Os resultados deste estudo apontam que existe correlação inversamente proporcional entre a gravidade da IU e a função sexual nas idosas incontinentes. Algumas idosas apresentaram ambas as disfunções, independentemente de serem ativas fisicamente.

Houve diferença na contração muscular perineal entre idosas com e sem DSF. No entanto, não foram encontradas diferenças significativas entre os grupos com e sem IU na contração muscular perineal e na função sexual. Porém, idosas com IU apresentaram tendência a resultados inferiores nessas variáveis, em relação às idosas sem IU.

Esses resultados reforçam a importância da avaliação dos MAP, orientação e prescrição individual de exercícios, atuação multiprofissional para idosas com IU e/ou DSF, além da participação conjunta com educadores físicos, fisioterapeutas, médicos entre outros profissionais da saúde.

\section{REFERÊNCIAS}

1. Castilho EA, Goldbaum M. Chronic noncommunicable diseases and population surveys. Rev Saúde Pública. 2017;51(Suppl 1). doi: 10.1590/S1518-8787.201705100suplled

2. Urinary incontinence in women. Female Pelvic Med Reconstr Surg. 2015;21(6):304-14. doi: 10.1097/SPV.0000000000000231

3. Aniuliene R, Aniulis P, Steibliene V. Risk factors and types of urinary incontinence among middle-aged and older male and female primary care patients in Kaunas region of Lithuania: cross sectional study. Urol J. 2016;12(1):2552-61.

4. Wolpe RE, Zomkowski K, Silva FP, Queiroz AP, Sperandio FF. Prevalence of female sexual dysfunction in Brazil: a systematic review. Eur J Obstet Gynecol Reprod Biol. 2017;211:26-32. doi: 10.1016/j.ejogrb.2017.01.018

5. Bezerra LRPS, Vasconcelos JA Neto, Vasconcelos CTM, Karbage SAL, Lima AC, Frota IPR, et al. Prevalence of unreported bowel symptoms in women with pelvic floor dysfunction and the impact on their quality of life. Int Urogynecol J. 2014;25(7):927-33. doi: 10.1007/s00192-013-2317-2
6. Rogers RG, Pauls RN, Thakar R, Morin M, Kuhn A, Petri E, et al. An International Urogynecological Association (IUGA)/ International Continence Society (ICS) joint report on the terminology for the assessment of sexual health of women with pelvic floor dysfunction. Int Urogynecol J. 2018;29(5):647-66. doi: 10.1007/s00192-018-3603-9

7. Tamanini JTN, Lebrão ML, Duarte YAO, Santos JLF, Laurenti R. Analysis of the prevalence of and factors associated with urinary incontinence among elderly people in the municipality of São Paulo, Brazil: SABE Study (health, wellbeing and aging). Cad Saúde Pública. 2009;25(8):1756-62. doi: 10.1590/ S0102-311X2009000800011

8. Chisholm L, Delpe S, Priest T, Reynolds WS. Physical activity and stress incontinence in women. Curr Bladder Dysfunct Rep. 2019 Sep;14(3):174-9. doi: 10.1007/s11884-019-00519-6

9. Virtuoso JF, Mazo GZ, Menezes EC. Incontinência urinária e função muscular perineal em idosas praticantes e nãopraticantes de atividade física regular. Rev Bras Fisioter. 2011;15(4):310-7. doi: 10.1590/S1413-35552011005000014

10. Virtuoso JF, Menezes EC, Mazo GZ. Effect of weight training with pelvic floor muscle training in elderly women with urinary incontinence. Res Q Exerc Sport. 2019;90(2):141-50. doi: 10.1080/02701367.2019.1571674

11. Tamanini JT, Dambros M, D’Ancona CAL, Palma PCR, Netto NR Jr. Validation of the "International Consultation on Incontinence Questionnaire: short form" (ICIQ-SF) for Portuguese. Rev Saúde Pública. 2004;38(3):438-44. doi: 10.1590/S0034-89102004000300015

12. Thiel RRC, Dambros M, Palma PCR, Thiel M, Riccetto CLZ, Ramos MF. Tradução para português, adaptação cultural e validação do Female Sexual Function Index. Rev Bras Ginecol Obstet. 2008;30(10):504-10. doi: 10.1590/S0100-72032008001000005

13. Laycock J, Jerwood D. Pelvic floor muscle assessment: the perfect scheme. Physiotherapy. 2001;87(12):631-42. doi: 10.1016/ S0031-9406(05)61108-X

14. Zeleke BM, Bell RJ, Billah B, Davis SR. Symptomatic pelvic floor disorders in community-dwelling older Australian women. Maturitas. 2016;85:34-41. doi: 10.1016/j.maturitas.2015.12.012

15. Virtuoso JF, Menezes EC, Mazo GZ. Fatores de risco para incontinência urinária em mulheres idosas praticantes de exercícios físicos. Rev Bras Ginecol Obstet. 2015;37(2):82-6. doi: 10.1590/SO100-720320140005040

16. Li T, Zhang YJ, Zhang HL, Ding XH, Yu ZJ, Lu S. Prevalence and risk factors of stress urinary incontinence among perimenopausal women and Its influence on daily life in women with sexual desire problem. Curr Med Sci. 2019;39(4):615-21. doi: 10.1007/s11596-019-2082-7

17. Zizzi PT, Trevisan KF, Leister N, Cruz CS, Riesco MLG. Women's pelvic floor muscle strength and urinary and anal incontinence after childbirth: a cross-sectional study. Rev Esc Enferm USP. 2017;51(10):1-8. doi: 10.1590/S1980-220X2016209903214

18. Hollinshead WH. Anatomia. 4a ed. Rio de Janeiro: Interlivros; 1991.

19. Bodner-Adler B, Alarab M, Ruiz-Zapata AM, Latthe P. Effectiveness of hormones in postmenopausal pelvic floor dysfunction: International Urogynecology Association research and development: committee opinion. Int Urogynecol J. 2020;31(8)1577-82. doi: 10.1007/s00192-019-04070-0 
20. Celik DB, Beji NK, Yalcin O. Sexual function in women after urinary incontinence and/or pelvic organ prolapse surgery. J Clin Nurs. 2014;23(17-18):2637-48. doi: 10.1111/jocn.12496

21. Su CC, Sun BYC, Jiann BP. Association of urinary incontinence and sexual function in women. Int J Urol. 2015;22(1):109-13. doi: 10.1111/iju.12610

22. Nilsson M, Lalos $\mathrm{O}$, Lindkvist $\mathrm{H}$, Lalos A. How do urinary incontinence and urgency affect women's sexual life? Acta Obstet Gynecol Scand. 2011;90(6):621-8. doi: 10.1111/j.1600-0412.2011.01120.x
23. Jha S, Ammenbal M, Metwally M. Impact of incontinence surgery on sexual function: a systematic review and meta-analysis. J Sex Med. 2012;9(1):34-43. doi: 10.1111/j.1743-6109.2011.02366.x

24. Visser E, Bock GH, Berger MY, Dekker JH. Impact of urinary incontinence on sexual functioning in community-dwelling older women. J Sex Med. 2014;11(7):1757-65. doi: 10.1111/ jsm.12544

25. Preda A, Moreira S. Stress urinary incontinence and female sexual dysfunction: the role of pelvic floor rehabilitation. Acta Med Port. 2019;32(11):721-6. doi: 10.20344/amp.12012 